\title{
Multilinguales
}

\section{L'enseignement du kabyle durant la période coloniale et la méthode directe : l'exemple des manuels de Boulifa}

The teaching of the kabyle during the colonial period and the direct method: the example of Boulifa manuals

\section{Nadia Berdous et Claude Cortier}

\section{(2penEdition}

Journals

Édition électronique

URL : https://journals.openedition.org/multilinguales/4352

DOI : $10.4000 /$ multilinguales. 4352

ISSN : 2335-1853

\section{Éditeur}

Université Abderrahmane Mira - Bejaia

\section{Référence électronique}

Nadia Berdous et Claude Cortier, «L'enseignement du kabyle durant la période coloniale et la méthode directe : l'exemple des manuels de Boulifa », Multilinguales [En ligne], 12 | 2020, mis en ligne le 14 février 2020, consulté le 30 juin 2021. URL : http://journals.openedition.org/multilinguales/4352 ; DOI : https://doi.org/10.4000/multilinguales.4352

Ce document a été généré automatiquement le 30 juin 2021.

\section{cc)}

Multilinguales est mise à disposition selon les termes de la Licence Creative Commons Attribution -

Pas d'Utilisation Commerciale - Pas de Modification 4.0 International 


\title{
L'enseignement du kabyle durant la période coloniale et la méthode directe : l'exemple des manuels de Boulifa
}

\author{
The teaching of the kabyle during the colonial period and the direct method: the \\ example of Boulifa manuals
}

Nadia Berdous et Claude Cortier

Comme la plupart des méthodologies d'enseignement, la méthodologie directe s'est élaborée en fonction de nouveaux besoins sociaux, alors suscités par la révolution industrielle mais aussi en réaction à la méthodologie traditionnelle qui accordait une place prépondérante à la traduction et qui nécessitait l'acquisition de connaissances grammaticales et culturelles importantes. A la fin du XIXe et au début du XXe siècle, face à l'extension du commerce et de l'industrie et à l'internationalisation des échanges, se développe sous l'impulsion des phonéticiens (Puren, 1988, Galazzi, 1991) un important mouvement réformateur de l'enseignement des langues vivantes, d'abord en Allemagne et en Scandinavie puis en France au début du XXe siècle :

On appelle méthodologie directe la méthode utilisée en Allemagne et en France vers la fin du XIXème siècle et le début du XXème siècle. Elle s'est également plus ou moins répandue aux Etats-Unis. En France l'expression "méthode directe" apparaît pour la première fois dans la Circulaire du 15 novembre 1901, qui l'opposait systématiquement à la méthodologie traditionnelle de grammaire-traduction en raison de son principe direct. Dans cette circulaire, on oblige pour la première fois tous les professeurs de langue étrangère à utiliser une méthodologie unique [...] (Rodriguez Seara, Ana)

Progressivement, se répand fortement l'idée que le but principal de l'enseignement des langues consiste d'abord à apprendre à les parler, et seulement ensuite à les écrire, et que leur connaissance pratique doit prévaloir sur l'acquisition d'une culture littéraire. C'est pourquoi, la méthode directe (désormais MD) a été officiellement imposée dans l'enseignement primaire et secondaire français par les instructions ministérielles de 
1902, et par extension, sous l'impulsion du ministre Jules Ferry, dans les colonies et protectorats (Vigner, 2015 ; Cortier et al., 2016). En Algérie, elle sera intégrée aux programmes d'enseignement d'abord pour le français et ensuite à l'enseignement des langues parlées pour les Européens,

Contestée par les tenants du bilinguisme (Machuel, 1885), elle évoluera dans la plupart des régions de France ou sous-domination française, très rapidement vers des méthodologies mixtes (Verny \& Lieutard, 2007), conservant des aspects traditionnels, tout en introduisant l'enseignement de l'oral, comme nous allons le voir également dans les manuels élaborés pour le kabyle par Boulifa.

Dans le présent article, nous nous intéresserons à l'enseignement du kabyle, comme langue étrangère, avec la méthode directe. Nous ferons une lecture descriptive analytique des deux manuels d'Amar Said Boulifa. L'objectif principal de cette étude est de voir comment Boulifa a exploité une méthodologie d'enseignement, conçue principalement pour les langues à large diffusion, comme le français, l'anglais... enseignées dans des cadres scolaires officiels, ayant une longue tradition écrite, pour l'enseignement du kabyle, langue orale et confinée dans des espaces domestiques et régionaux.

\section{1- L'enseignement du kabyle durant la période coloniale et la méthode directe}

L'extension considérable de l'espace colonial français à la fin du XIXe siècle, conduit les autorités françaises à créer en 1894 un ministère des Colonies, chargé de donner cohérence à l'action politique et administrative de la France dans ses colonies. La même année est fondée l'École coloniale dont la mission sera de former les administrateurs et les magistrats ayant à intervenir dans les colonies. (Vigner, 2015).

Une politique éducative va se mettre en place, en Algérie, et permettra l'ouverture d'une chaire pour l'enseignement du berbère, du kabyle et de l'arabe algérien. Jusqu'à cette période, la connaissance du berbère était resté l'apanage d'un milieu très restreint, la production savante étant mal adaptée aux objectifs pratiques des instituteurs et des administrateurs (Selles, 2013). Cet enseignement sera accompagné par des primes conséquentes pour encourager les fonctionnaires français et européens à suivre ces enseignements :

L'école normale inaugurée à Alger en 1866, destinée à former de futurs directeurs d'écoles arabes-françaises, reste après le tournant politique de 1870 un pôle actif d'enseignement de l'arabe et du kabyle, et ce avant même la mise en place, au début des années 1890, d'une section spéciale pour les élèves-maîtres se destinant à l'enseignement indigène. (Messaoudi, $2013: 84$ )

Selon l'ouvrage Notions de kabyle (Mohamed El Hocine et Plault, 1960), plusieurs instances délivraient des diplômes, brevets et certificats de berbère (tamazight) : la faculté d'Alger, l'École Pratiques d'Études Berbères et l'École Nationale des Langues Orientales. Chaker, quant à lui, parle surtout de deux écoles qui ont formé des générations de berbérisants : l'Ecole normale de Bouzaréah et l'Ecole supérieure des lettres qui allait devenir la Faculté des lettres d'Alger (Chaker, 1996 :01). Certains Algériens, particulièrement des Kabyles, ont suivi ces formations et les dispenseront par la suite, à l'instar d'Amar Said Boulifa et de Belkassem Ben Sedira. 
Cet enseignement avait d'abord un objectif utilitaire, il concernait surtout les instituteurs qui allaient entrer en contact direct avec les kabylophones ou berbérophones. Puis, il a été élargi à tous les fonctionnaires qui voulaient bénéficier de la prime accordée aux titulaires de diplôme de langue berbère et/ou de langue kabyle, notamment pour ceux et celles qui voulaient accéder à certains postes administratifs, pour lesquels la maitrise $d u$ berbère et kabyle était une condition sine qua non d'admission.

6 Les manuels d'enseignement de langue kabyle, durant la période coloniale, ont été d'une manière générale élaborés avec des méthodologies conçues pour l'enseignement/ apprentissage des langues étrangères préconisées en France : «Il s'agissait de donner aux jeunes diplômés, dont les instituteurs surtout, une formation spéciale qui devait les préparer à enseigner dans les écoles kabyles ou ailleurs en Algérie.» (Berdous, 2017: 228). Cela a toutefois permis un travail linguistique important et la production d'outils didactiques spécifiques :

Le renouvellement des modes d'enseignement des langues vivantes avec le primat de la méthode directe, l'institution de primes pour les fonctionnaires berbérophones (1881) et de certifications universitaires (avec un brevet et un diplôme délivrés par l'école des Lettres d'Alger à partir de 1885) suscitent la réalisation de nouveaux ouvrages [...] (Selles, $2013: 142$ ).

Les différentes méthodologies de cet enseignement se sont inscrites dans une continuité identique à celle des langues vivantes où la méthodologie traditionnelle a d'abord été dominante, puis vint la méthode directe, "explicitement recommandée par les nouveaux programmes arrêtés par le recteur d'Alger en 1898 " (Messaoudi, 1913 : 98), avec plusieurs manuels pour l'arabe et, pour le kabyle, les manuels de Boulifa. Vers les années 60 , la didactique du kabyle, langue étrangère, s'est enrichie avec le manuel Tiziwuccen1 qui relève de la méthodologie audio-visuelle en vogue à l'époque en France.

Soulignons cependant, que le grand changement dans l'enseignement du kabyle durant la période coloniale fut amorcé par les manuels de Boulifa qui orienta nettement l'enseignement vers la pratique orale de la langue :

$\mathrm{Au}$ cours de 'deuxième année', l'élève étant suffisamment initié aux choses théoriques de la langue, sera appelé à se livrer à des exercices beaucoup plus pratiques et, partant, moins monotones. Basée sur la conversation, la méthode n'a, en effet, d'autre but que d'habituer l'élève à la manœuvre pratique, à l'emploi, avec ses propres moyens, des connaissances acquises pour l'amener graduellement, à s'initier à la gymnastique spéciale du langage. (Boulifa, 1913 : VIII).

L'accent mis sur l'enseignement de l'oral dans le deuxième manuel de Boulifa l'inscrit dans l'esprit de la MD, mais dans un deuxième temps, à la différence des autres méthodes de ce courant : «Ce principe direct en effet ne se réfère pas seulement dans l'esprit de ses promoteurs à un enseignement des mots étrangers sans passer par l'intermédiaire de leur équivalent français, mais aussi à celui de la langue orale sans passer par l'intermédiaire de sa forme écrite [...] » (Puren, 1988 : 95). 


\section{L'enseignement d'Amar Said Boulifa et la méthode directe}

\section{Un berbérisant prolixe}

Né vers 1865 à Adni (Fort National, Grande Kabylie), orphelin très jeune, Amar Said Boulifa fut élevé par son oncle maternel qui le scolarisa à Tamazirt, la toute première école ouverte en Kabylie en 1875, un établissement qui formera de nombreuses générations au savoir moderne. Instituteur formé à l'Ecole Normale de Bouzareah dans les années 1890, il devint ensuite répétiteur de berbère à la même école, puis en 1901, à la Faculté des lettres d'Alger :

Boulifa a été le prototype de l'instituteur et de l'érudit kabyle de formation française, totalement acquis aux idéaux de l'école républicaine française, à ses objectifs affichés de promotion et d'égalité, mais au même temps profondément fier de sa culture et de sa langue d'origine auxquelles il consacrera toute sa vie (CRB-

Centre de Recherche Berbère : 02).

Il prit sa retraite en 1929 et mourut en 1931 à Alger où il fut inhumé.

Boulifa fut un berbérisant prolixe, il s'est intéressé d'abord à l'enseignement de la langue kabyle, pour lequel il a élaboré deux manuels avec la méthode directe. Le premier manuel est intitulé "Première année de langue kabyle» (Boulifa, 1897), le deuxième est intitulé "Langue kabyle-cours de deuxième année, étude linguistique et sociologique sur la Kabylie de Djurdjura» (Boulifa, 1913). Ces deux manuels sont complémentaires et assurent un enseignement cohérent étalé sur deux années.

Il s'intéressa aussi à la littérature et à l'histoire. Il a écrit plusieurs ouvrages utiles pour la connaissance du kabyle dont les plus connus sont: Recueil de poésies kabyles (texte zouaoua)2, précédé d'une Etude sur la femme kabyle et d'une notice sur le chant kabyle (airs de musique), Le Djurdjura à travers l'histoire (depuis l'Antiquité jusqu'à 1830), Organisation et indépendance des Zouaoua (Grande Kabylie)...

\section{Un didacticien innovant}

10 L'enseignement du kabyle durant la période coloniale a connu diverses méthodes comme nous l'avons souligné, toutefois, Boulifa, premier des instituteurs kabyles à s'inscrire dans ce renouveau méthodologique, s'est affirmé comme une figure incontournable de l'élaboration des manuels :

«Ses manuels scolaires constituent une référence incontestable pour ses contemporains et les générations ultérieures. Ils mettent en œuvre la méthode directe à partir de textes directement puisés auprès de locuteurs kabyles » (Selles, $2013: 142)$.

Boulifa explique dans la préface de son premier manuel les objectifs de sa méthode :

Apprendre à parler et écrire correctement le kabyle, guider et encourager ceux qui veulent s'adonner à l'étude de cette langue, les mettre dans le moins de temps possible à même de converser avec les Indigènes, tel est le but que je me suis proposé en rédigeant ce modeste ouvrage qui n'est que le résumé des leçons que j'ai l'honneur de faire devant les élèves de la Section spéciale de l'Ecole normale d'Alger-Bouzareah depuis décembre 1891(Boulifa, 1897, Préface : 1). 
11 Il insiste d'emblée sur la maitrise de l'oral, car le kabyle est une langue parlée, que l'on doit enseigner comme telle. Mais comme toute langue, elle a des règles qui lui sont propres :

Si l'on jette un coup d'œil sur la gradation des leçons et exercices, il semble de prime abord, que l'ordre logique et grammatical n'a pas été suivi, il faut rappeler que le kabyle, tout en étant une langue parlée, n'en a pas moins ses règles particulières... C'est pour obvier à ces difficultés que j'ai cru devoir suivre une méthode et un plan tout particulier (Boulifa, $1897: 2$ ).

Il affirme ensuite la nécessité d'une connaissance solide du vocabulaire et de la grammaire car il faut habituer dès le commencement l'élève ou l'apprenant à exprimer ses idées au moyen d'un vocabulaire étudié : "Pour arriver à une possession solide et pratiquement utilisable d'une langue il faut beaucoup de mots et assez de grammaire, (Boulifa, 1913 : VIII). Il souligne plus loin :

Pour préparer le Kabylisant à la conversation, j'ai écarté ce genre de phrase que l'on ne trouve guère que dans les manuels et que l'on rencontre jamais dans la pratique ; je me suis efforcé de faire un choix judicieux de tournures et d'expressions qui sont d'un fréquent usage dans le langage courant (Boulifa, 1897).

Et puis Boulifa insiste, dans son premier manuel sur l'accent mis sur le volet oral de la langue et non pas le volet de l'écrit, ce qui le rapproche, mais sans l'inscrire totalement, dans l'esprit de la méthode directe.

Par ailleurs, et c'est la grande originalité de sa production, Boulifa a élaboré lui-même les textes supports pour la lecture, il s'est inspiré de la vie quotidienne des Kabyles, de leurs us et coutumes, comme il le souligne dans la préface du cours de deuxième année :

«nous estimons donc que l'étude de la vie pratique de ceux dont on veut connaître la langue, est là, toute trouvée pour nous servir de base et pour nous procurer toutes les matières voulues, devant composer notre deuxième année.» (Boulifa, 1913 : IX).

Dans le manuel de première année, il commence par un cours introductif sur l'alphabet et les adaptations qu'il a jugé nécessaires pour enseigner le kabyle. Il insiste sur la prononciation (voir les Remarques en annexe $n^{\circ} 1$ ). Chaque leçon se termine par des exercices de prononciation comme le recommandent explicitement les instructions officielles de $1902^{1}$ :

Tous les efforts du professeur devront tendre à obtenir dès le début une prononciation et une accentuation exactes. Afin d'y parvenir, il prononcera luimême les vocables lentement et en séparant les syllabes, les fera répéter tantôt par un seul élève, tantôt par plusieurs, tantôt par l'ensemble de la classe, jusqu'à ce qu'il ait obtenu une reproduction exacte des sons qu'il a émis.

Toutefois, il s'est éloigné de l'un des principes les plus courants de la méthode directe, en recourant, comme le montre la page en annexe $n^{\circ} 1$, à la langue française. Seuls les exemples qui accompagnent les cours sont donnés en langue kabyle. Cela peut s'expliquer par le fait qu'il s'agit d'un manuel que l'apprenant pouvait être amené à travailler seul.

Les leçons de vocabulaire se résument à la mémorisation d'un nombre de mots présentés dans l'ordre naturel :

Ces mots ne sont pas pris au hasard; leur classification est faite d'après l'ordre naturel : ainsi le mot izzem, lion n'est évidemment pas classé dans la catégorie des mots où se trouve par exemple afalkou, faucon (oiseau) mais la catégorie des quadrupèdes (animaux sauvages) (Boulifa, 1897). 
Boulifa, s'alignant sur le principe de la MD a privilégié l'apprentissage du vocabulaire courant et de proximité : la première leçon de vocabulaire porte sur les mots qui désignent d'abord la classe, puis l'école, la maison... La méthode directe préconise de commencer par les mots du vocabulaire concret qui désignent des réalités palpables, d'abord des objets que l'enseignant peut montrer dans l'environnement proche, puis progressivement et selon une gradation, on introduit des univers plus lointains, puis d'autres mots plus abstraits qui seront explicités à partir des mots déjà connus. (Voir la liste des mots à prononcer figurant dans la page annexe $\mathrm{n}^{\circ} 2$ où se retrouvent la salle de classe, le corps humain, les animaux, etc.)

Comme nous le constatons, Boulifa a respecté certains principes de la méthode directe mais il en a rejeté d'autres. Il n'a pas suivi comme un dogme les principes de la MD, la langue française est toujours présente dans le manuel de la première année, bien que ce soit comme l'a montré Puren "l'interdiction de l'utilisation de la langue maternelle des élèves qui fonde réellement la MD en tant méthodologie nouvelle» (Puren, 1988 : 122). Cependant, les exercices de grammaire qui consistent à travailler directement sur la langue elle même, sont récurrents dans son premier manuel, mais également les exercices de thèmes et versions qui renvoient directement à méthode traditionnelle : «dans lesquels les règles sont appliquées et les mots appris trouvent un emploi immédiat. » (Boulifa, 1897).

Dans son deuxième manuel de langue kabyle, Cours de deuxième année, étude linguistique et sociologique sur la Kabylie du Djurdjura, Boulifa orienta sa méthode vers la pratique orale de la langue, il élimina toutes les leçons de grammaire, considérées comme acquises durant la première année, se rapprochant ainsi des principes de la méthode directe, puisque :

Ce principe direct en effet ne se réfère pas seulement dans l'esprit de ses promoteurs à un enseignement des mots étrangers sans passer par l'intermédiaire de leur équivalent français, mais aussi à celui de la langue orale sans passer par l'intermédiaire de sa forme écrite. (Puren, 1988 : 95).

Comme l'a montré Puren (1988), le noyau dur de la MD est constitué de trois méthodes : méthode directe, méthode active et méthode orale, une terminologie que nous retrouvons dans le discours de Boulifa : "Basée sur la conversation, la méthode n'a, en effet, d'autre but que d'habituer l'élève à la manœuvre pratique, à l'emploi, avec ses propres moyens, des connaissances acquises pour l'amener graduellement à s'initier à la gymnastique spéciale du langage. (Boulifa, 1913 : VIII).

\section{L'adaptation de la méthode directe au contexte linguistique et culturel kabyle}

Boulifa a souligné dans sa préface l'importance de l'enseignement du vocabulaire pour l'apprentissage d'une langue étrangère. Pour son manuel Méthode de langue kabyle - cours de deuxième année il préconise un enseignement basé sur la connaissance de la Kabylie (sa culture, son organisation sociale, ses coutumes...).

Pour ce faire, il a imaginé des échanges (des dialogues et de longs textes) entre un instituteur étranger et les différents membres de la société kabyle (agriculteur, artisan, femme...). Le personnage principal est

« un Français, un instituteur, ami des habitants dont il instruit les enfants. Ayant le vif désir de s'instruire, il provoquera les questions, il interrogera les gens au milieu desquels il vit (...)» (Boulifa, $1913: \mathrm{X})$ 
Ces échanges qui portent sur le pays kabyle (dans toutes ses dimensions) permettent au personnage nommé opportunément Afransis de connaitre et de se familiariser avec les coutumes de ce pays et ses habitants.

Ce sont des textes grâce auxquels Amar Said Boulifa a su insérer les mots du lexique à acquérir dans leurs contextes d'emploi, et pas seulement comme une liste de mots à apprendre par cœur. L'exemple ci-dessous montre un texte qui met en scène l'instituteur français dialoguant avec un villageois.

La méthode directe mise en œuvre par Boulifa pour l'enseignement du kabyle est originale par son adaptation au contexte kabyle. En effet, pour cet instituteur kabyle connaitre une langue, c'est :

Connaitre la mentalité, l'esprit, le génie de ceux qui parlent cette langue; or, la mentalité d'un groupe quelconque d'individus, ne peut être pénétrée, saisie, comprise que par une étude approfondie, une connaissance exacte de leur caractère et de leurs mœurs. C'est là, pensons-nous, l'esprit de la méthode directe (Boulifa, 1913 : VIII)

C'est une définition qui donne à cet enseignement une dimension culturelle et sociale et l'éloigne de l'objectif pratique assigné à l'enseignement de la langue étrangère dans le cadre de la méthodologie directe qui se voulait un nouvel outil de maitrise effective de la langue comme simple instrument de communication, parti pris contre lequel se sont élevés beaucoup de pédagogues (Cortier \& Puren, 2008, Costa, 2009).

Bien qu'il souligne explicitement la primauté de l'oral sur l'écrit, Boulifa maintient le besoin de la grammaire pour une maitrise solide de la langue. Là encore, il ne s'éloigne pas complètement de la méthode traditionnelle. On peut supposer retrouver dans ce souci d'un enseignement théorique, une volonté de grammatisation, d'élaboration linguistique, en même temps que didactique, inhérent à la mise en place de l'enseignement d'une langue orale.

21 Et pour conclure, Boulifa a proposé, dans ses méthodes et manuels, une conception originale et propre à lui de la méthode directe, il ne s'est pas emprisonné dans les principes qui ont guidé son application en France, comme le non recours à la langue maternelle du locuteur, en première année notamment, année où les élèves - maitres prennent contact avec le kabyle pour la première fois. On sait que ce principe a été critiqué par nombre de pédagogues et didacticiens et que son application dans les régions allophones a été largement contestée en France et même en Tunisie, où le Directeur de l'enseignement public Louis Machuel préconisera un enseignement «bilingue » pour ne pas interrompre le développement intellectuel des enfants. La Revue pédagogique se fera d'ailleurs l'écho de ces contestations, plus particulièrement entre 1888 et 1894, comme le soulignent Cortier et al. (2016).

Boulifa a également adapté cette méthode conçue principalement pour l'enseignement des langues étrangères à large diffusion pour enseigner une langue orale et minorée. Dans ses manuels, il n'y pas d'illustrations comme c'est l'usage dans les manuels élaborés avec la méthode directe. Son public est constitué d'élèves-maitres, jeunes adultes qui pouvaient se passer facilement des illustrations pour saisir le sens des leçons. Il a, cependant, exploité des textes culturels "anthropologiques" comme supports didactiques, dans une vision moderne de l'enseignement des langues, où l'enseignement institue l'importance de la tradition orale :

» Dans les différents processus de l'élaboration didactique des langues minoritaires, mêlant ainsi très souvent les avancées les plus significatives en matière de 
méthodes et les savoir- faire ancestraux de la transmission orale » (Di Meglio, 2015 :

6)

Il ne s'est pas limité dans son enseignement au seul aspect linguistique, il a intégré la dimension socio-culturelle de la langue enseignée, une démarche construite visant l'acquisition de compétences socio-culturelles: "où l'enseignant ou le formateur doit devenir, par excellence, pour l'apprenant, le relais nécessaire, le médiateur privilégié, vers une autre culture, celle dont il enseigne la langue» (Gohard-Radenkovic, $2004: 11)$, une approche moderne de l'enseignement des langues étrangères.

\section{BIBLIOGRAPHIE}

La lecture descriptive et critique que nous avons proposée des manuels de Boulifa, à la lumière des études plus récentes portant sur les manuels de langue berbère (Selles, 2013 ; Berdous, 2017 : 199), montre des points de vue contrastés sur la façon de concevoir la méthode directe. Boulifa ne l'avait ni contestée ni adoptée telle qu'elle. Il l'avait adaptée au contexte qui était le sien prenant en considération un public de jeunes adultes, le statut du kabyle, l'environnement socioculturel et les tensions socio-politiques de l'époque.

ABROUS, N., L'enseignement du berbère : analyse comparée Algérie/Maroc, thèse de doctorat soutenue sous la direction de Salem Chaker, Aix-Marseille, 2017.

ALLAIN, M., et BROUSSE, L., Tizi-wwuccen. Méthode multimédia de langue tamazight, Edition FCNAFA, 2012.

BERDOUS, N., La question du kabyle en Algérie. Individuation sociolinguistique et processus d'élaboration didactique, Thèse de doctorat soutenue sous la direction d'Alain Di Meglio, Université de Corte, 2017.

BOULIFA, A. S., Une première année de langue kabyle (dialecte zouaoua), A l'usage des candidats à la prime et au brevet de kabyle, Alger, Jourdan, 1897, 2e édition, 1910.

BOULIFA, A. S, Méthode de langue kabyle (cours de deuxième année). Étude linguistique, sociologique sur la Kabylie du Djurdjura. Texte zouaoua, Alger, Jourdan, Alger, 1913.

CENTRE DE RECHERCHE BERBERE, « BOULIFA si Amar- ou-Saïd (1865-1931) : le grand précurseur berbérisant », voir https://www.centrederechercheberbere.fr/tl_files/doc- pdf/Boulifa.pdf consulté le 13/04/2019

CHAKER, S., « Enseignement », in Gabriel Camps (dir.), Volumes n 17, Edisud, 1996, mis en ligne le 01 juin 2011, consulté le 01 mai 2019. 17, sur le site : http://journals.openedition.org/ encyclopedieberbere/2157

CHAKER, S., « Documents sur les précurseurs. Deux instituteurs kabyles : A. S. Boulifa et M. S. Lechani » In : Revue de l'Occident musulman et de la Méditerranée, $\mathrm{n}^{\circ} 44,1987$, consulté sur le site : www.persee.fr/doc/remmm_0035-1474_1987_num_44_1_2159 le [14/10/2019]

CHEMAKH, S., « La standardisation du kabyle », Communication au Colloque sur l'aménagement linguistique, Tizi-Ouzou, Avril 2000, Consulté le 13.10.2019 sur [https://linguistiqueamazighe.blog4ever.com/articles/mes-articles-publias] 
CILIANU - LASCU, C., « Analyse des différentes méthodologies et méthodes d'enseignement/ apprentissage des langues étrangères ", dans : Dialogos/18, 2008, consulté sur le site : www.romanice.ase.ro/dialogos/18/01_Cilianu-Analise.pdf (le 22/08/2017)

CORTIER, C., DI MEGLIO, A., OTTAVI, P., « Langues minorées dans l'ensemble méditerranéen. Sociodidactique et convergences contextuelles par l'exemple du corse ", Dans Messaoudi, L., Rispail, M., Cahiers de linguistique, 2016-41-1, Des langues minoritaires dans l'espace plurilingue francophone. Mélanges offerts à Ahmed Boukous, 2016, pp. 11-40.

CORTIER, C., PUREN, L., « Français et langues régionales et/ou minoritaires : une mise en convergence difficultueuse ", dans : Dimensions socioculturelles de la didactique du français, Repères, 38,2008 , pp. 63-80.

COSTA, J., « Les langues régionales au cœur des travaux sur la variation langagière : cent ans de publications de l'INRP », Trema, 31, 2009, pp. 7-14.

DI MEGLIO, A., « Elaboration didactique d'une langue minorée : le corse. Problématique de l'enseignement du corse par l'approche sociolinguistique de documents didactiques entre 1974 et 1994 », Université de Corte, 1997.

DI MEGLIO, A., « D'une identité réactive à une identité créative : aspects patrimoniaux de la transposition didactique de la langue corse ", dans Vivre du patrimoine, un nouveau modèle de développement, textes réunis par J-M. Furtet A.Fazi, Editions L'Harmattan, 2010.

DI MEGLIO, A., « Didactique des langues minoritaires et enjeux de légitimation », in Guide pour la recherche en didactique des langues et cultures (approches contextualisées), P. Blanchet/P. Chardenet (Dir.), AUF, Prefics Rennes 2, Editions des archives contemporaines, 2015.

GOHARD-RADENKOVIC, A., Communiquer en langue étrangère, de compétences culturelles vers des compétences linguistiques, Editions PETERLAN, 2004 (2ème édition), consulté le 19/10/2019 sur le site : https://books.google.dz > books

MACHUEL, L., Méthode de lecture et de langage à l'usage des étrangers de nos colonies (traduite en langue annamite, arabe et italienne), Armand Colin, 1885, réédition 1896.

MEKSEM, Z., « Enseignement de tamazight en Algérie : des précurseurs jusqu'à aujourd'hui » communication à l'IRCAM- Maroc 4 -5 décembre 2003.

MEKSEM, Z., Pour une sociodidactique de la langue amazigue : approche textuelle, thèse de doctorat sous la direction de Marielle Rispail, Université Stendhal (Grenoble), 2007

MESSAOUDI, A., « Progrès de la science, développement de l'enseignement secondaire et affirmation d'une « méthode directe » (1871-1930) », dans : LARZUL, S., (DIR.) ; MESSAOUDI, A., (dir.), Manuels d'arabe d'hier et d'aujourd'hui : France et Maghreb, XIXe-XXIe siècle, Éditions de la Bibliothèque nationale de France, Paris. 2013.

PUREN, C., « Histoire de la didactique des Langues - Cultures et Histoires des idées », https:// www.aplv- languesmodernes.org/docrestreint.api/ 619/1a28d6213c6ee2815eacf132b18ff5ba206cb59d/pdf/PUREN_Modeles_ideologiques_DLC.pdf, consulté le 27/06/2019.

PUREN, C., Histoire des méthodologies de l'enseignement des langues, Editions Clé internationale, Paris, 1988.

RODRIGUEZ SEARA, A., « L'évolution des méthodologies dans l'enseignement du français langue étrangère depuis la méthodologie traditionnelle jusqu'à nos jours. ", consulté le 19/10/2019 sur le site : https://qinnova.uned.es > archivos_publicos > qweb_paginas 
SELLES, M., «Les manuels de berbère publiés en France et en Algérie (XVIIIe-XXe siècle) : d'une production orientaliste à l'affirmation d'une identité postcoloniale », dans :LARZUL, S., (DIR.) ; MESSAOUDI, A., Manuels d'arabe d'hier et d'aujourd'hui : France et Maghreb, XIXe-XXIe siècle, Éditions de la Bibliothèque nationale de France, Paris. 2013

SI AHMED, M., et PLAULT, M., Notions de kabyle, (grammaire, exercices, textes dialogues) : Aït Iraten, Aït Aggouacha du Nord, à l'usage des candidats à l'examen pour la prime de $4 \mathrm{e}$ catégorie, Lyon, 1960.

VERNY, M - J., et LIEUTARD, H., L'école française et les langues régionales XIXe et XXe siècles, Presses universitaires de la Méditerranée, Montpellier, 2007.

VIGNER, G., «L'École Jules-Ferry, école normale de l'enseignement colonial : une formation pour apprendre à enseigner dans les colonies (1902-1912) », Documents pour l'histoire du français langue étrangère ou seconde [En ligne], 55 | 2015, mis en ligne le 01 janvier 2018, consulté le 10 mai 2019. URL : http://journals.openedition.org/dhfles/4273

\section{NOTES}

1. - La méthode directe a été officiellement imposée en 1902 mais elle été employée et recommandée la fin des années 1890. Voir (Messaoudi, 1913 : 98)

\section{RÉSUMÉS}

Cet article propose une lecture descriptive analytique de l'enseignement du kabyle durant la période coloniale. Nous nous sommes intéressées aux manuels de Boulifa élaborés avec la méthode directe, imposée en France par les instructions ministérielles de 1902. En Algérie, elle sera intégrée aux programmes d'enseignement pour le français et à l'enseignement des langues parlées comme le kabyle.

This article offers a descriptive analytical reading of Kabyle teaching during the colonial period. We were interested in the Boulifa textbooks developed with the MD imposed in France by the ministerial instructions of 1901. In Algeria, it will be integrated into the teaching programs for French and then to the orals languages as Kabyle.

\section{INDEX}

Mots-clés : Méthode directe, enseignement du kabyle, manuels d'enseignements, programmes d'enseignement, période coloniale

Keywords : Direct method, Kabyle education, teaching manuals, teaching programs, colonial period 


\section{AUTEURS}

NADIA BERDOUS

Université de Bouira, Algérie

\section{CLAUDE CORTIER}

Université de Lyon, France 